\title{
LOCALIZED SALIENT REGION-BASED OBJECT DETECTION AND TRACKING
}

\author{
Supreeth $\mathrm{H} \mathrm{S} \mathrm{G}^{1 *}$ and Chandrashekar M Patil ${ }^{2}$ \\ ${ }^{1}$ Department of Electronics and Communication, SJB Institute of Technology, \\ India \\ ${ }^{2}$ Department of Electronics and Communication, Vidyavardhaka College of \\ Engineering, India \\ ${ }^{1}$ supreeth751@gmail.com, ${ }^{2}$ patilcm@gmail.com
}

\begin{abstract}
This paper proposes a method to embed multiple features of an image for object localization and salient region for object detection and tracking. Initially, the object location is obtained using ground truth and features are extracted. Subsequently, these features are used to find the potential area of an object and this area is used to identify a salient region. The pixels color and gradients are used as feature matrix and saliency maps are generated using the visual cue like the local contrast. The salient region detection has proved its application in object recognition and segmentation. The combination of feature matrix and saliency map enables the proposed tracker to be robust. The proposed method efficiently localizes the object even when there are scaling, rotation and illumination changes. The experiments are conducted using publicly available Visual object tracking (VOT) 2016 dataset which consists of many challenging video sequences and the proposed method provided competitive results when compared to many state-of-the-art methods which is evaluated using the Visual object tracking toolkit.
\end{abstract}

Keywords—object detection, salient region, object tracking, localization

\section{INTRODUCTION}

Visual object tracking is a challenging task in computer vision because of the complexity involved in locating an object in every frame. The fundamental importance of visual tracking lies in the areas of surveillance, human behavior analysis, humancomputer interaction, driverless vehicles and so on. Once the tracker is initialized by providing the coordinates of the object to be tracked, the tracker has to predict the location or the state of the same object in the subsequent frames accurately. Many factors affect the tracking accuracy like object scaling, rotation, illumination variations, occlusion and fast motion. Many algorithms have been proposed to deal with the mentioned problems. The existing methods can be classified into two groups: generative methods and discriminative methods.

The generative trackers define the object to be tracked in the form of appearance model, and the entire image is searched for the image region with the best score in the successive frames. The results of searching depend on how good the appearance model is defined or designed. Some of the generative tracking methods are mean shift tracker [1], multi-task tracker (MTT) [2], a low-rank sparse tracker [3] and L1-min tracker [4]. The discriminative tracking methods are like binary classification where it exploits the difference between background and foreground. Background information plays an

Received: May 10, 2019

Reviewed: August 21, 2019

Accepted: September 16, 2019 
important role in identifying the target object but it has some challenges like the classification of boundaries and sample size. Support vector tracking, multiple instance learning (MIL) and deep learning methods are the different types of discriminative tracking methods.

The proposed method is based on tracking-by-detection method and data association. The tracking-by-detection is one of the mainstream methods of tracking objects proposed in [5]. This method is used to distinguish the target object from the background and it is a type of discriminative method of tracking objects. Data association is continuously identifying the same object in subsequent frames. For the data association, the featurebased technique is used [6]. The features like color values (R, G, B) and gradient values are used. This vector is utilized in order to search for the object in the top-down approach. The multiple features are fused to make tracking robust and versatile whereas low dimensional matrix makes computation cost modest. In the proposed method a motion model is defined which assumes that the object to be tracked will appear in proximity in the next frame.

The proposed method first extracts the features of the object defined by the bounding box values and the same features are used to identify the object in the next frame by searching the neighborhood. After searching the high confidence score regions will be selected that region is the salient region in which the object is localized. The advantage of considering the salient region is the object can be easily identified from the background in a bottom-up approach using local contrast. The salient region consists of the object to be tracked. The proposed method is capable of tracking any object without restriction of the size, shape, orientation, and color.

The rest of the paper is organized as follows: the review of some related work based on feature descriptors and salient region in Section 2. The proposed methodology is presented in Section 3. Discussion of results and evaluation is presented in Section 4 and the paper finishes in section 5 with conclusions.

\section{RELATED WORK}

This section reviews the previous literature on feature descriptors for object tracking and salient object detection. Many region descriptors have been proposed in the literature for different applications like object recognition and tracking. In [7] a color based method is proposed in which color is one of the widely used image feature in object tracking for its effectiveness but color histogram is not robust to illumination variations and pose changes. Pixel intensities hardly represent the appearance of a scene; they do not capture image attributes and therefore cannot be able to characterize what an image represents. Research works have also focused on the development of simple yet potential region descriptors that can consider multiple aspects like color, texture, and gradients.

The salient object detection algorithms are categorized into bottom-up and top-down approaches. Low level information like color, intensity and spatial distances are used to build saliency maps in bottom-up approach. Recent bottom-up approaches use local, regional and global contrast to find the saliency. In [8] a first central-surrounded based approach is proposed which uses color, intensity, and orientation in multiple scales for visual saliency detection. A method to compute saliency map using regional contrast along with global contrast extraction in the entire image is proposed in [9], where the image is in Lab color space. Without the prior knowledge, the bottom-up saliency detection methods can work well and provide useful information. The bottom-up saliency detection methods fail when they encounter complex backgrounds.

The top-down saliency detection is a task-driven technology, and it calls for high-level cues like earlier understanding of the challenge, emotions and expectations. A method which includes high-level information such as humans, faces, and texts which attract more gazes, and convey essential information in an image when compared to other low- 
level visual information is proposed in [10]. A unified Bayesian approach for task-driven visual attention is proposed in [11] it includes information like the global context of a scene and previous locations which are integrated over time to predict the next location. The limitation of the existing top-down saliency detection methods is: harsh conditions limit the practicability and applicability and also the computational complexity is high.

Global contrast based methods are gaining interests and providing promising results. These methods consider the global statistics of the entire image for processing. In [12] a method is proposed which will utilize the global motion contrast by considering the keypoint correspondences and geometric transformations of consecutive images followed by the pixel-level global color contrast in each image.

\section{PROPOSED METHODOLOGY}

In this section, the proposed localized salient-region based object detection and tracking (LSOT) is demonstrated via feature extraction, saliency map generation. The block diagram of the proposed method is shown in Figure 1. The proposed LSOT tracker is initialized using the first frame and bounding box values from the ground truth to get the object. Multiple features of the object are extracted and a single feature matrix is generated. In the subsequent frames, previous object location and its neighborhood are searched for potential regions of the object. The localized potential region so obtained is used to generate saliency map. The location of the salient object that is found is used to update the tracker.

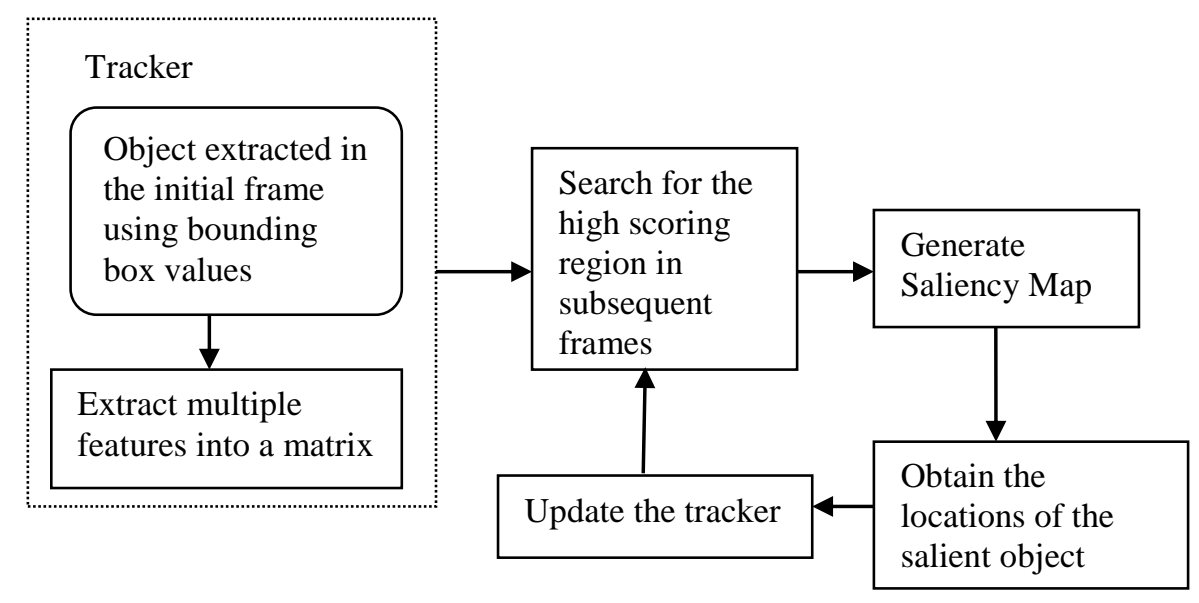

Fig. 1 The proposed localized salient-region based object detection and tracking method

\subsection{FEATURE EXTRACTION AND COSINE SIMILARITY}

This section describes the extraction of features in a single video frame. In every location of object, seven different pixel-level features are extracted, which includes normalized color intensities of red, green and blue channel, first order derivates of gray scale intensity in $\mathrm{X}$ and $\mathrm{Y}$ direction and second order derivates of gray scale intensity in $\mathrm{X}$ and $\mathrm{Y}$ direction as in [13]. The representation of visual information can be efficiently done using this way. The pixel representation is given in Eq. (1).

$$
f=\left[R, G, B, \frac{\partial I}{\partial x}, \frac{\partial I}{\partial y}, \frac{\partial^{2} I}{\partial x^{2}}, \frac{\partial^{2} I}{\partial y^{2}}\right]
$$

Where R, G, and B are the red, green and blue intensity channels and I is the corresponding grayscale intensity image. 
In the initial frame, the features of the object to be tracked are extracted and this feature is used to compare the potential regions in subsequent frames using cosine distance. The high scoring regions usually belong to in and around the object of interest and those regions are selected to identify the salient object which filters out the unwanted region.

The cosine distance between two vectors $\mathrm{a}$ and $\mathrm{b}$ is defined as

$$
d=1-\frac{a \square b}{\|a\|\|b\|}
$$

Where is the dot product of the vectors a and $b$.

Since the search of regions in a frame is localised and not exhaustive the algorithm works fast and real-time in nature.

\subsection{SALIENCY MAP GENERATION}

In the pre-processing stage, the input image is segmented into abstract regions, where each region is called as superpixels which are created using the simple linear iterative clustering (SLIC) algorithm is proposed in [14]. Each abstract region is associated with a single node, where each node is connected only to its neighbouring nodes. The image is converted to lab color space and superpixels are generated by clustering pixels. For each input frame, $\mathrm{N}$ number of clusters is generated.

The integration of different salient maps is proposed in [15]. So in the proposed method salient feature map is generated using multiple feature maps. In [16] a method of generating visual saliency by using a multi-feature and multi-scale representation of the image and fusing local contrast features is proposed.

In the proposed method saliency map generation takes place in three different stages: (1) Selection of salient features from frames (2) The saliency map generation by local contrast diffusion and (3) Integration of the saliency maps generated.

The obtained localized object region from a frame using a feature search is used to divide it into a set of feature maps. Then the color, luminance, and local orientation information are extracted from these maps. The luminance feature map is termed as I, color feature map is termed as CRG, CBY and orientation feature map as $\mathrm{O}$ and the respective equations is as follows:

$$
\begin{aligned}
& I=\frac{r+g+b}{3} \\
& C_{R G}=\frac{r-g}{\max (r, g, b)} \\
& C_{B Y}=\frac{b-\min (r, g)}{\max (r, g, b)} \\
& O(\theta)=\left\|I G_{o}(\theta)\right\|+\left\|I G_{\pi / 2}(\theta)\right\|
\end{aligned}
$$

Where $r$ is red channel value, $g$ is green channel value and $b$ is the blue channel value of individual image pixels. I denote the luminance, $\mathrm{G}($.$) is the Gabor filter function and$ the local orientation is $\theta$, where $\theta=\left\{0^{\circ}, 45^{\circ}, 90^{\circ}, 135^{\circ}\right\}$. The saliency feature map SFM is given as

$$
S_{F M}=\left\|I_{k}+C_{k}+O_{k}\right\|
$$

Where $\mathrm{I}_{\mathrm{k}}, \mathrm{C}_{\mathrm{k}}, \mathrm{O}_{\mathrm{k}}$ represents the luminance, color, and orientation of the $\mathrm{k}^{\text {th }}$ frame of the video sequence. 
The local contrast methods can improve the appearance of foreground objects based on the contrast of the neighbours of the object in question. The saliency map using spatial distribution measure is also determined to fuse it with saliency map generated using multiple features $S_{\mathrm{FM}}$.

To improve the reliability of the object detection and the general quality of salient region segmentation, the measure of distribution is required for a superpixel $\mathrm{P}_{\mathrm{i}}$ with respect to the centroid of saliency map $\mathrm{S}_{\mathrm{FM}}$.

$$
d m(i)=\frac{\sum_{j=1}^{N} s_{i j} \cdot n_{j} \cdot\left\|c_{j}-\mu_{s}\right\|}{\sum_{j=1}^{N} s_{i j} \cdot n_{j}}
$$

where $S_{i j}$ is the similarity between a pair of superpixels $\left(P_{i}\right.$ and $\left.P_{j}\right)$ according to their spatial distance, $\mathrm{nj}$ is the total number of pixels belong to the superpixel $\mathrm{P}_{\mathrm{j}}, \mathrm{C}_{\mathrm{j}}$ is the centroid of the superpixel $\mathrm{P}_{\mathrm{j}}, \mu_{s}=\left[\mu_{s}^{x}, \mu_{s}^{y}\right]$ is the centroid of saliency map $\mathrm{S}_{\mathrm{FM}}$. The similarity function $S_{\mathrm{ij}}$ and the centroid $\mu_{\mathrm{s}}$ are defined as

$$
S_{i j}=e^{-\frac{\left\|c_{i}-c_{j}\right\|}{\sigma^{2}}}
$$

Where $\sigma$ is the constant

$$
\mu_{s}^{x}=\frac{\sum_{i=1}^{N} S_{F M}(i) \cdot n_{i} \cdot c_{i}^{x}}{\sum_{i=1}^{N} S_{F M}(i) \cdot n_{i}}
$$

And

$$
\mu_{s}^{y}=\frac{\sum_{i=1}^{N} S_{F M}(i) \cdot n_{i} \cdot c_{i}^{y}}{\sum_{i=1}^{N} S_{F M}(i) \cdot n_{i}}
$$

Finally, the salient feature map generated using multiple features $S_{\mathrm{FM}}$ and the distribution measure $\mathrm{dm}$ is fused together to get the final saliency map $\mathrm{S}$.

$$
S=S_{F M}+d m
$$

The final salient region obtained is binarized to get the exact location of the object. The location/coordinate value is used to update the tracker in every frame.

\section{EXPERIMENTAL EVALUATION}

The proposed method is evaluated on the Visual Object Tracking VOT2016 [17] dataset, it consists of 60 videos sequences including 20,000 images. The dataset has many challenges in every sequence which includes occlusion, background clutter, scaling, illumination variation, and object transformation. The evaluation is carried out using MATLAB software running on Intel Core i5 processor. The VOT evaluation mainly consists of two parameters they are accuracy and robustness. The measure of bounding box overlap of the estimated rectangle and the ground truth is called as accuracy. The number of tracking failures in each sequence is called as robustness. 


\section{Original Frame Localised object Salient object Tracking output}

(a)
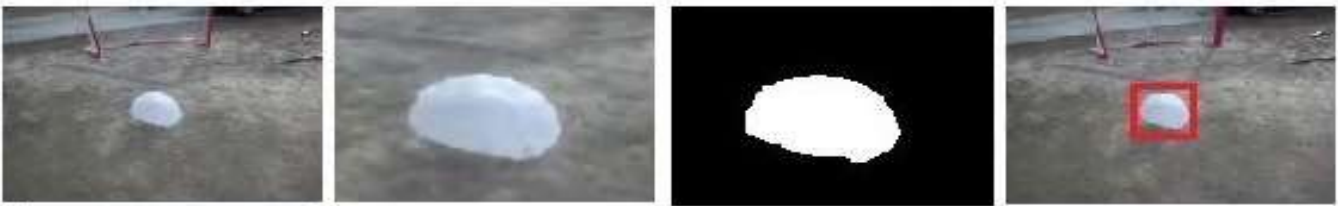

(b)
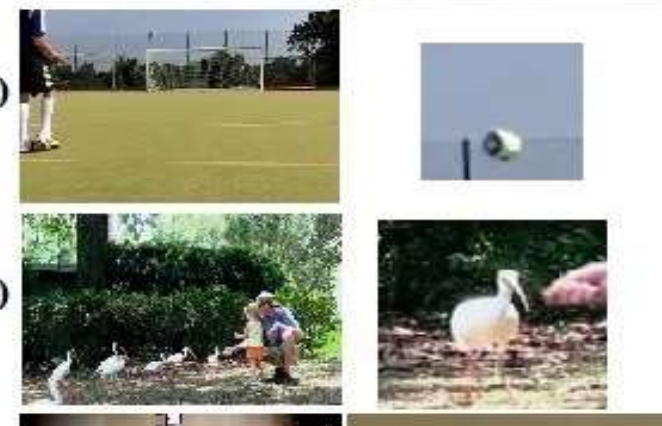

(d)

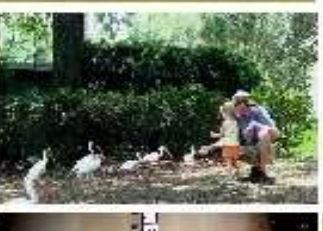

(c)
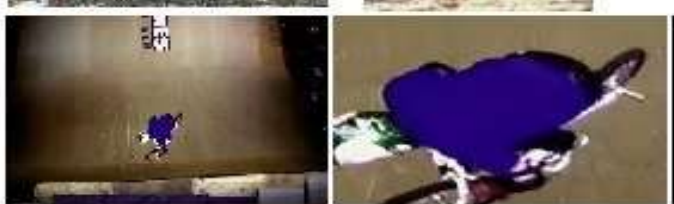

(e)

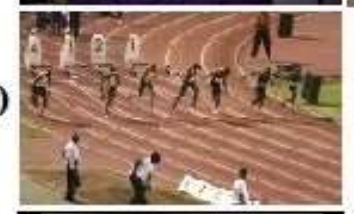

(f)
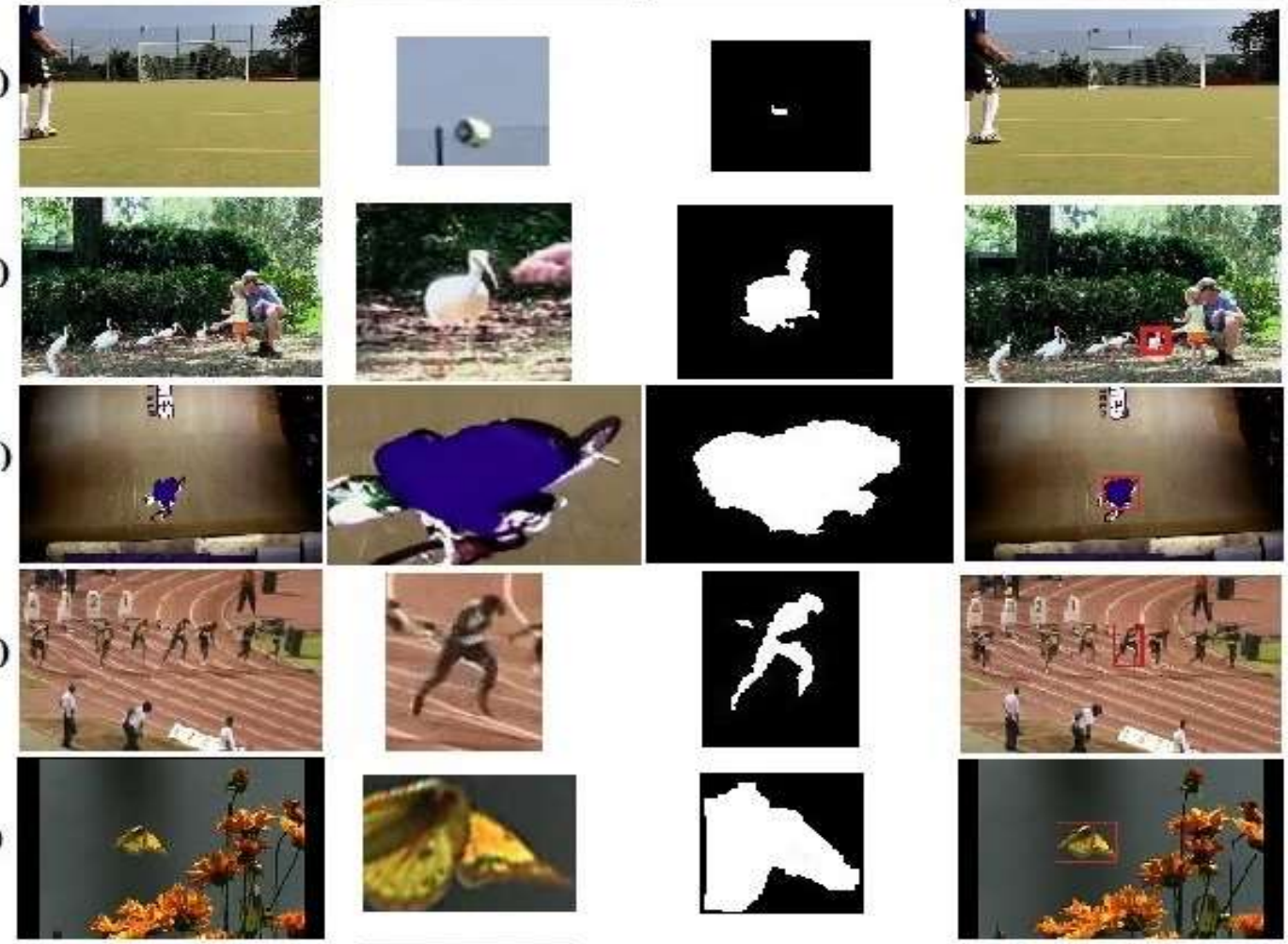

(g)

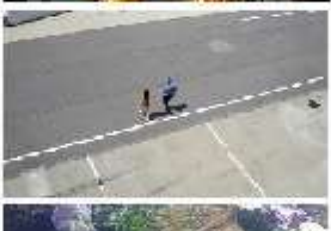

(h)
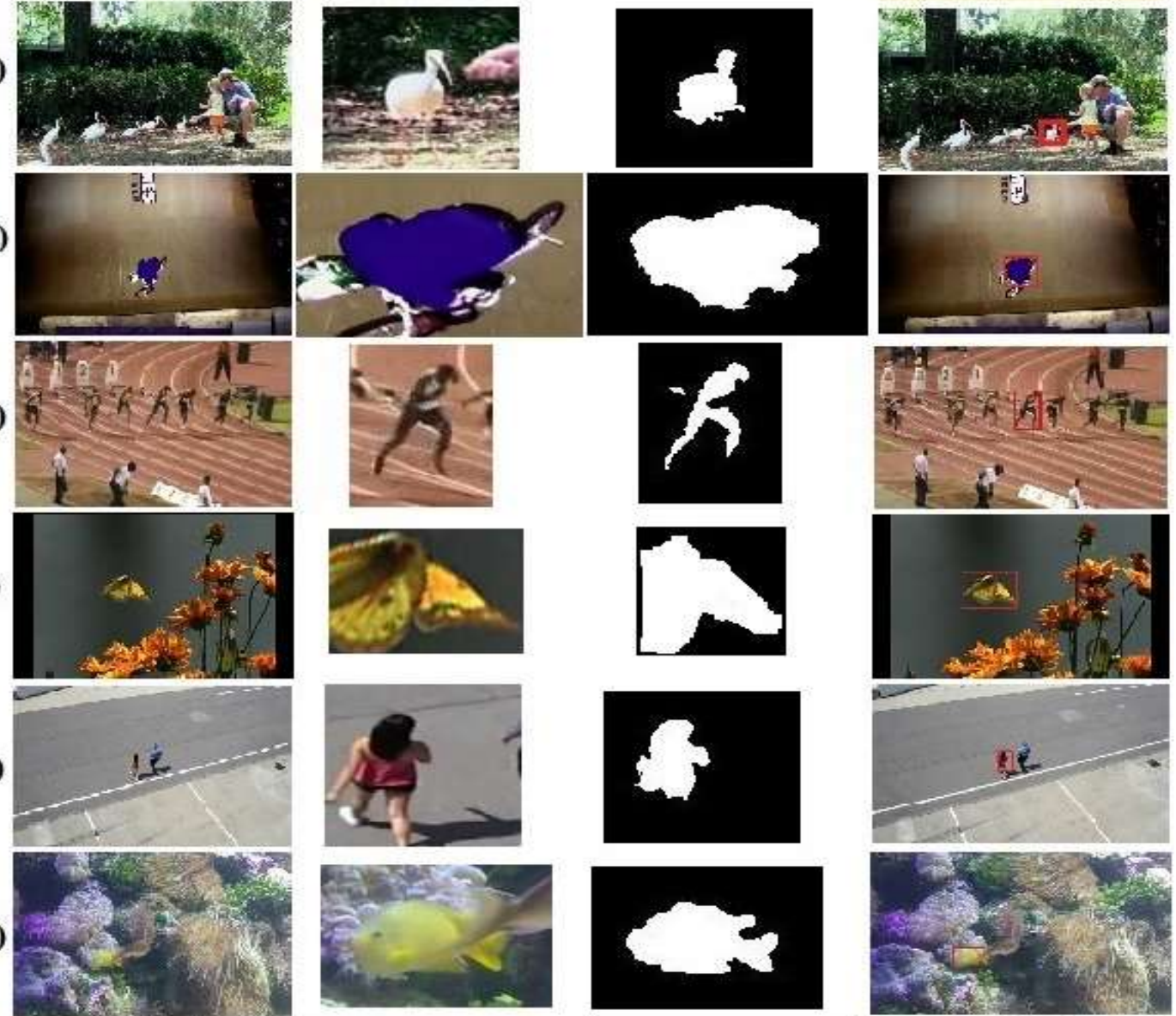

(i)
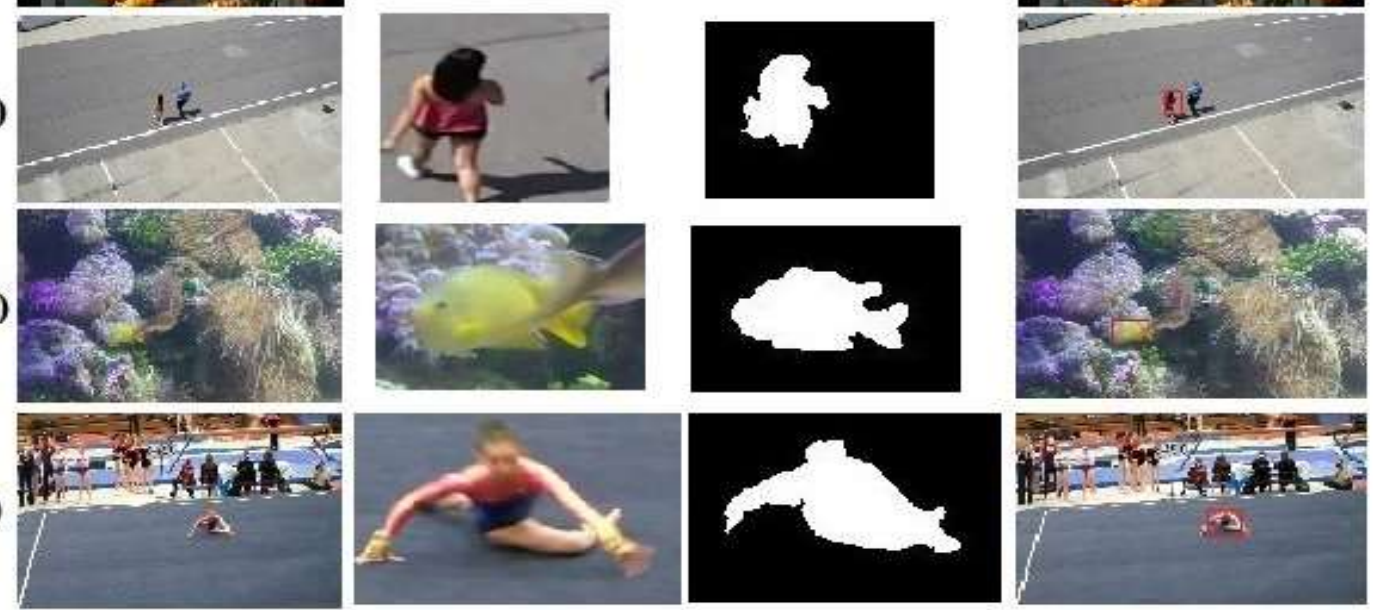


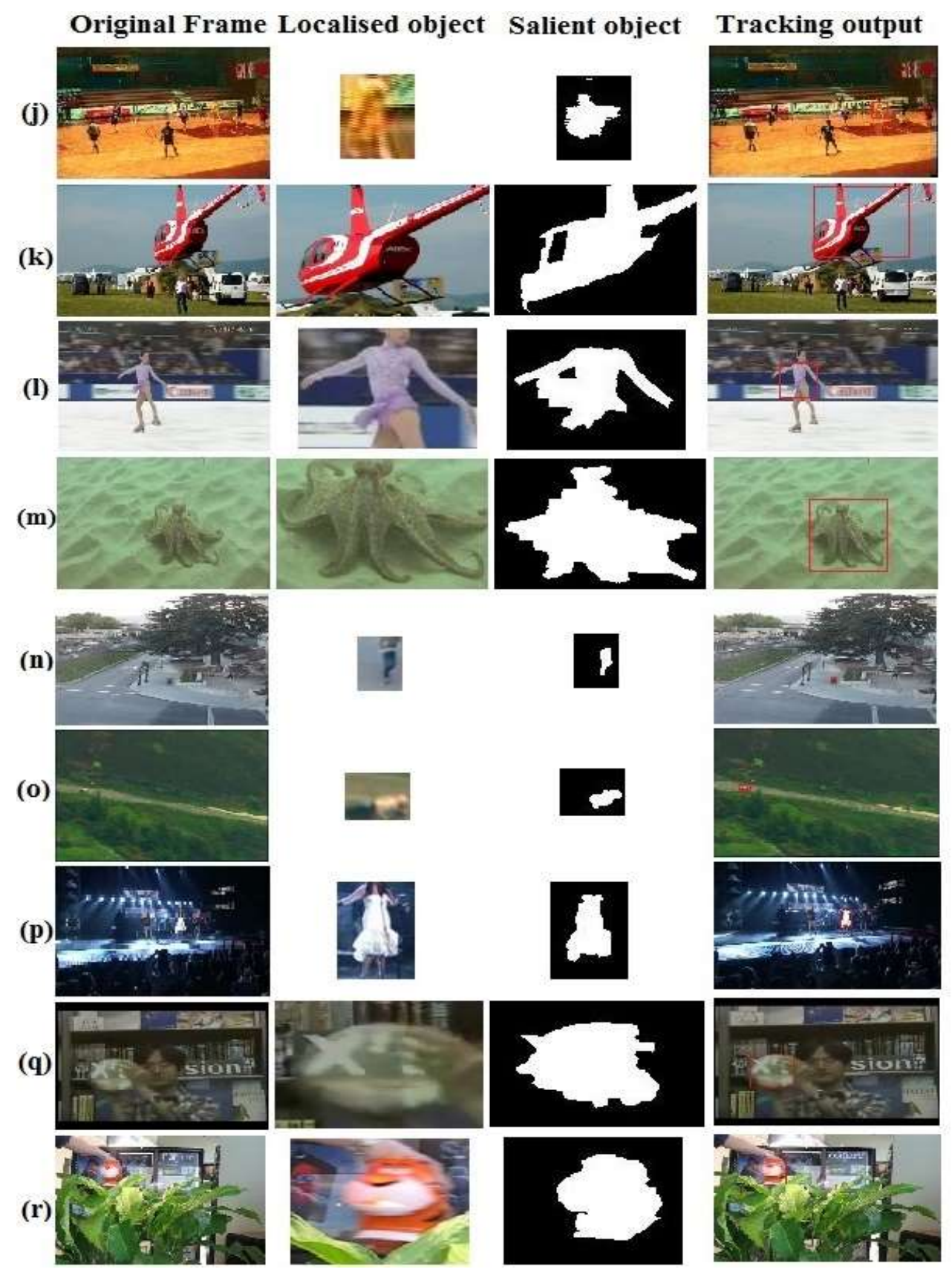

Fig. 2 The output of the proposed method for different sequences (a) bag (b) ball2 (c) birds2 (d) bmx (e) bolt2 (f) butterfly (g) crossing (h) fish3 (i) gymnastics4 (j) handball2 (k) helicopter (1) iceskater1 (m) octopus (n) pedestrian2 (o) road (p) singer1 (q) sphere (r) tiger

In the first frame of every sequence, the VOT evaluation toolkit initializes the tracker to the ground truth bounding box value of the first frame. If there is a failure in tracking the toolkit automatically reinitializes the tracker after a few frames. The high score of accuracy is good because it is the weighted mean of the overlap whereas low robustness score is good because it is the weighted average of failure count in tracking. The final ranking of the tracker is based on the Expected Average Overlap (EAO) which is the average of overlap ratio of all sequences in the dataset. The normalized speed of the tracker is provided by Equivalent Filter Operations (EFO). The total computation time is divided by EFO which reduces the hardware dependencies. The proposed method is 
compared with seven state-of-the-art methods reported in VOT committee. The output of the proposed method is shown in Figure 2.

Table I. BASELINE RESULTS FOR VOT 2016 DATASET

\begin{tabular}{|c|c|c|c|c|}
\hline Tracker & EAO & Accuracy & Robustness & EFO \\
\hline LSOT & 0.32 & 0.548 & 33.36 & 5.42 \\
\hline DSST & 0.18 & 0.532 & 50.01 & 9.71 \\
\hline KCF & 0.19 & 0.504 & 42.41 & 15.74 \\
\hline DAT & 0.22 & 0.454 & 34.03 & 9.69 \\
\hline DNT & 0.28 & 0.503 & 22.79 & 1.08 \\
\hline SRBT & 0.29 & 0.474 & 24.44 & 2.22 \\
\hline MDNet_N & 0.26 & 0.542 & 23.54 & 0.51 \\
\hline deepMKCF & 0.23 & 0.542 & 29.66 & 1.14 \\
\hline
\end{tabular}

The proposed tracker LSOT is tested on VOT2016 dataset and the obtained results are compared with other tracking methods. The LSOT achieved higher EAO results in baseline and unsupervised category. The baseline results provided from VOT toolkit are presented in Table I. The LSOT tracker out performs the state-of-the-art trackers in EAO and accuracy. The proposed method is compared with two correlation filter based methods (KCF [18], DSST [19]), three CNN feature based methods (MDNet_N [20], deepMKCF [21], DNT [22]) and color based methods (DAT [23], SRBT [24]). The robustness measure of DSST method stands first whereas EFO of KCF is high when compared to others.

The EAO results comparison of different methods is shown in Figure 3. The unsupervised results comparison is presented in Table II. The unsupervised overlap graph for different methods is shown in Figure 4.

Table II. UNSUPERVISED RESULTS FOR VOT 2016 DATASET

\begin{tabular}{|c|c|c|}
\hline Tracker & Overlap & EFO \\
\hline LSOT & 0.481429 & 5.62 \\
\hline DSST & 0.344286 & 8.66 \\
\hline KCF & 0.324286 & 14.88 \\
\hline DAT & 0.288571 & 13.65 \\
\hline DNT & 0.412857 & 1.13 \\
\hline SRBT & 0.321429 & 2.32 \\
\hline MDNet_N & 0.461429 & 0.57 \\
\hline deepMKCF & 0.41 & 1.39 \\
\hline
\end{tabular}



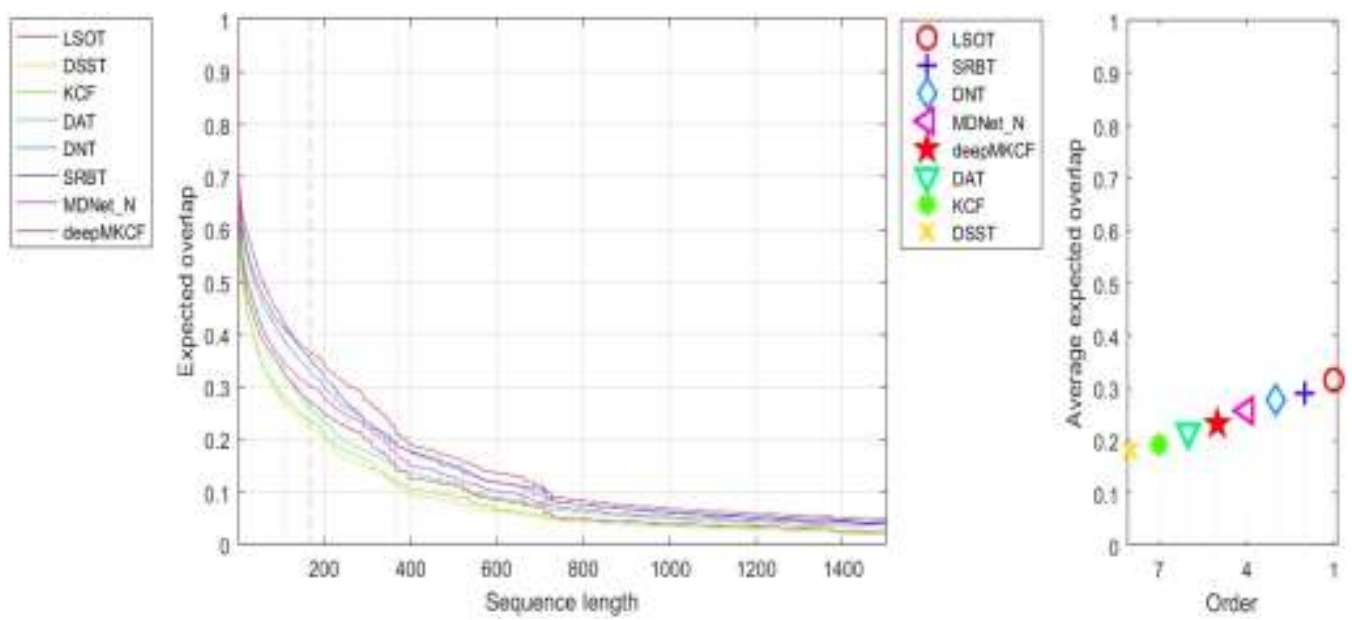

Fig. 3 The baseline EAO graph for VOT 2016 challenge

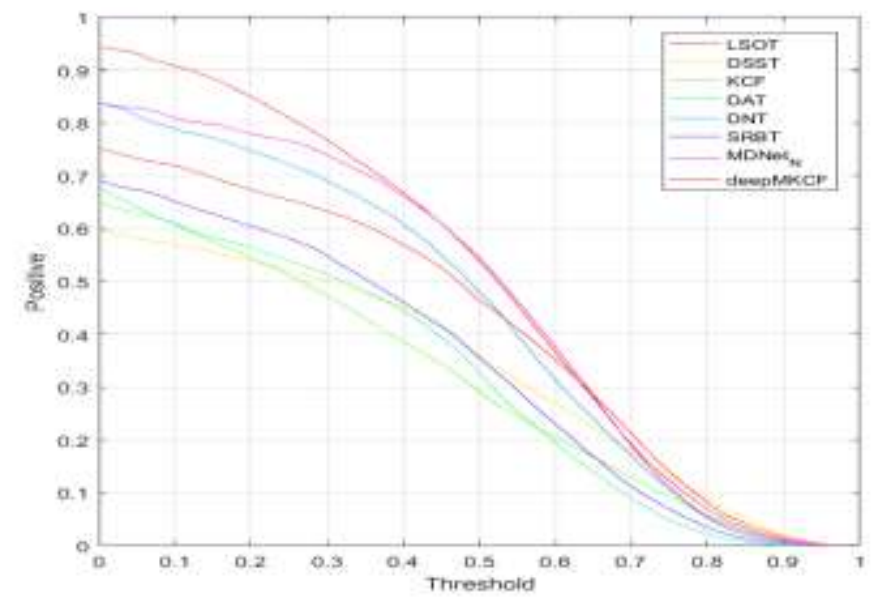

Fig. 4 The unsupervised overlap graph of different methods

\section{CONCLUSION}

This paper proposed localized salient region-based object detection and tracking (LSOT) which uses multiple features to localize the object in the entire frame and finds the salient object from the obtained region. The multiple feature saliency map and spatial distribution measure are fused to improve the accuracy of salient object detection. This method works on the potential region of the object which reduces the time consumption. The proposed method was evaluated using a publicly available dataset which consists of many challenging sequences. The evaluation result proves that the proposed method achieved higher EAO than the existing state-of-the-art trackers.

\section{REFERENCES}

[1] Comaniciu, D., Ramesh, V. and Meer, P. "Kernel-based object tracking” IEEE Transactions on Pattern Analysis and Machine Intelligence 25.5 (2003): 564-577.

[2] Zhang, T., Ghanem, B., and Liu, S. "Robust visual tracking via multi-task sparse learning" Computer vision and pattern recognition (2012): 2042- 2049.

[3] Zhang, T., Ghanem, B. and Liu, S. "Low-rank sparse learning for robust visual tracking" European conference on computer vision, (2012): 470- 484.

[4] Mei, X. and Ling, H. "Robust visual tracking using 11 minimization" International conference on computer vision (2009): 1436-1443. 
[5] Kristan, M., Matas, J., Leonardis, A., Felsberg, M., Cehovin, L., Fernandez, G., Vojir, T., Hager, G., Nebehay, G. and Pflugfelder, R. "The visual object tracking vot2015 challenge results", IEEE International Conference on Computer Vision Workshops, Santiago, Chile, 7-13 December 2015, pp.123.

[6] Frintrop, S., Klodt, M., and Rome, E. "A real-time visual attention system using integral images" Fifth International Conference on Computer Vision Systems (ICVS), Bielefeld, Germany, 2007.

[7] Grove, T. D., Baker, K.D. and Tan, T. N. "Color based object tracking" Fourteenth International Conference on Pattern Recognition, Brisbane, Queensland, Australia, 2002.

[8] Itti, L., Koch, C. and Niebur, E., "A model of saliency-based visual attention for rapid scene analysis", IEEE Transactions on Pattern Analysis and Machine Intelligence 20.11 (1998): 1254-1259.

[9] Cheng, M. M., Zhang, G. X., Mitra, N. J., Huang, X. and Hu, S. M. "Global contrast based salient region detection" IEEE Conference on Computer Vision and Pattern Recognition, Colorado Springs, CO, USA, 20-25 June 2011, pp. 409-416.

[10] Judd, T., Ehinger, K. A., Durand, F. and Torralba, A. "Learning to predict where humans look" IEEE Twelfth International Conference on Computer Vision, Kyoto, Japan, 29 September - 2 October 2009, pp. 2106-2113.

[11] Ali, B., Dicky, N. and Itti, S. L. "Probabilistic Learning of Task-Specific Visual Attention" IEEE Conference on Computer Vision and Pattern Recognition, Providence, RI, USA, 16-21 June 2012, pp. 470-477.

[12] Zhai, Y. and Shah, M. "Visual attention detection in video sequences using spatiotemporal cues" Fourteenth ACM international conference on Multimedia, Santa Barbara, CA, USA, 23 - 27 October 2006, pp.815-824.

[13] Tuzel, O., Porikli, F. and Meer, P. "Region Covariance: A Fast Descriptor for Detection and Classification"Lecture Notes in Computer Science, Vol. 3952. Springer, Berlin, Heidelberg, 2006, pp. 589-600.

[14] Achanta, R., Shaji, A., Smith, K., Lucchi, A., Fua, P. and Susstrunk, S. "SLIC superpixels compared to state-of-the-art superpixel methods" IEEE Transactions on Pattern Analysis and Machine Intelligence, 34.11 (2012): 2274-2282.

[15] Zhou, L., Yang, Z., Yuan, Q., Zhou, Z. and Hu, D. "Salient Region Detection via Integrating DiffusionBased Compactness and Local Contrast" IEEE Transactions on Image Processing, 24.11 (2015): 33083320.

[16] Liu, Y., Yang, F., Zhong, C., Tao, Y., Dai, B. and Yin, M. "Visual tracking via salient feature extraction and sparse collaborative model" International Journal of Electronics and Communications, 87 (2018): 134-143.

[17] Kristan, M. et al. "The Visual Object Tracking VOT2016 Challenge Results" Computer Vision ECCV Workshops. Lecture Notes in Computer Science, Vol. 9914. Springer, Cham, 2016, pp. 777-823.

[18] Henriques, J. F., Caseiro, R., Martins, P. and Batista, J. "High speed tracking with kernelized correlation filters" IEEE Transactions on Pattern Analysis and Machine Intelligence, 37.3 (2015): 583-596.

[19] Danelljan, M., Hager, G., Khan, F. and Felsberg, M. "Accurate scale estimation for robust visual tracking" British Machine Vision Conference, Nottingham, 2014.

[20] Nam, H. and Han, B. "Learning multi-domain convolutional neural networks for visual tracking" IEEE Conference on Computer Vision and Pattern Recognition, Las Vegas, NV, USA, 27-30 June 2016, pp. 4293-4302.

[21] Tang, M. and Feng, J. "Multi-kernel correlation filter for visual tracking" IEEE International Conference on Computer Vision, Santiago, Chile, 7-13 December 2015, pp. 3038-3046.

[22] Chi, Z., Li, H., Lu, H. and Yang, M.H. "Dual Deep Network for Visual Tracking" IEEE Transactions on Image Processing, 26.4 (2017): 2005-2015.

[23] Possegger, H., Mauthner, T. and Bischof, H. "In defense of color-based model-free tracking" IEEE Conference on Computer Vision and Pattern Recognition, Boston, MA, USA, 7-12 June 2015, pp.21132120.

[24] Lee, H. and Kim, D. "Salient Region-Based Online Object Tracking" IEEE Winter Conference on Applications of Computer Vision, Lake Tahoe, NV, USA, 12-15 March 2018, pp. 1170-1177. 